\title{
Pipeline architectures of three-dimensional daubechies wavelet transform using hybrid method
}

\author{
Noor Huda Ja'afar', Afandi Ahmad ${ }^{2}$ \\ ${ }^{1}$ Section of Instrumentation and Control Engineering, Universiti Kuala Lumpur Malaysian Institute of Industrial \\ Technology, Malaysia \\ ${ }^{2}$ Department of Electrical and Electronic Engineering, Universiti Tun Hussein Onn Malaysia, Malaysia
}

\begin{tabular}{l} 
Article Info \\
\hline Article history: \\
Received Dec 14, 2018 \\
Revised Feb 15, 2019 \\
Accepted Mar 1, 2019 \\
\hline
\end{tabular}

\section{Keywords:}

DWT

FPGA

Hybrid method

Image compression

\begin{abstract}
The application of three-dimensional (3-D) medical image compression systems uses several building blocks for its computationally intensive algorithms to perform matrix transformation operations. Complexity in addressing large medical volumes data has resulted in vast challenges from a hardware implementation perspective. This paper presents an approach towards very-large-scale-integration (VLSI) implementation of 3-D Daubechies wavelet transform for medical image compression. Discrete wavelet transform (DWT) algorithm is used to design the proposed architectures with pipelined direct mapping technique. Hybrid method use a combination of hardware description language (HDL) and G-code, where this method provides an advantage compared to traditional method. The proposed pipelined architectures are deployed for adaptive transformation process of medical image compression applications. The soft IP core design was targeted on to Xilinx field programmable gate array (FPGA) single board RIO (sbRIO 9632). Results obtained for 3-D DWT architecture using Daubechies 4-tap (Daub4) implementation exhibits promising results in terms of area, power consumption and maximum frequency compared to Daubechies 6-tap (Daub6).
\end{abstract}

Copyright (C) 2019 Institute of Advanced Engineering and Science. All rights reserved.

\section{Corresponding Author:}

Noor Huda Ja'afar,

Section of Instrumentation and Control Engineering,

Universiti Kuala Lumpur Malaysian Institute of Industrial Technology,

81750 Bandar Seri Alam, Johor, Malaysia.

Email: noorhuda.jaafar@unikl.edu.my

\section{INTRODUCTION}

In the high-tech world, various types of medical tools such as electrocardiogram (ECG), electromyography (EMG), computed tomography (CT), positron emission tomography (PET), ultrasound (US), magnetic resonance imaging (MRI) and X-ray routinely used to diagnose and analyze illness inside human body [1-3]. In the context of medical algorithms, a massive amount of data to be processed has affected the organization of the memory units. It requires more space to store the data, as well as the intermediate data before being used for the next process. Moreover, most of the sub-disciplines of medical image processing are contributed to the matrix transformation operations [4-6]. Thus, efficient implementations of the medical imaging algorithms are very challenging. To get over these issues, the FPGAs are ideally suited for the hardware implementations and at the same time to achieve better performances includes speed, size and power [7].

A basic operation of image compression system involves three process, which are transformation, quantization and entropy coding. An array of input pixels will go through the first transformation process for having the transformed coefficients as the output. Then, the transformed coefficients are quantized to produce a finite number of levels. In addition, the entropy coding process is applied to the finite set of numbers to 
give an additional compression. In technical view, an image is containing of smooth and sharp variations. The smooth variation is the base of the image and represents the low-pass variation. On the other side, the sharp variation represents the high-pass variation and both variations are added together to gives a detailed image. In this context, the purpose of the transformation process is to separate the smooth and sharp variations.

A wavelet act as a mathematical tool to extract information from different kinds of data such as audio signals and images [8]. Comparing with Discrete Cosine Transform (DCT), DWT provides more advantages and excellent coding gain for image processing applications. The DWT use a frame-based computation concept which the image can be separated into many tiles where a larger tile size can avoid the blocking artifacts.

In general, there are two types of design methodology that can be used to synthesizes and implement the proposed architectures, which are traditional and hybrid methods. Both methods applied a similar procedure of programmed the Hardware Description Language (HDL) using Xilinx Integrated Software Environment (ISE). The traditional method is a basic design flows that use the HDL for FPGA synthesize and implementation. On the other hand, the hybrid method uses a combination of HDL and Graphical Programming (G-code), where this method provides an advantage compared to the traditional method.

In this paper, Daubechies wavelet transform architecture have been proposed and examined specifically for 3-D transform in medical image compression. The aim of this paper is to develop an efficient reconfigurable architecture of Daubechies wavelet transform using pipelined direct mapping with hybrid method. An evaluation in terms of area, power consumption and maximum frequency is also carried out to analyze the performance of the proposed architectures.

The rest of the paper is organized as follows. An overview of the related work is given in Section 2. Mathematical background for Daubechies wavelet transform is described in Section 3. Experimental results and analysis of are presented in Section 4. Concluding remarks and further potential ideas to be explored are given in Section 5.

\section{LITERATURE REVIEW}

The fundamental unit on computer is represented in a number of bits. For instance, an image containing 640×480 pixels (12-bit gray scale) needs more than 3 Megabits per second of storage. Therefore, to transmit this image via conventional phone lines with speed range of 56 Kilobits per second, it consumes more than one second [9]. For this reason, image compression is important to provide an efficient data storage and data transmission [10-12]. In general, the purpose of image compression is to reduce the size and blocking artifact of original image without degrading the quality of the image. Because of that, the development of an efficient image compression technique becomes the most challenging matter.

Moreover, compressing medical image is more challenging compared to non-medical image. This is because for medical images, the compression algorithms are complex and it should always be stored in lossless format even though sometimes lossy format is acceptable [10, 12]. In addition, medical images are extremely rich with information contents. Therefore, there is a real need for high-performance systems, whilst keeping architectures flexible to allow for quick upgradeability with real-time applications [4].

However, most of the existing works carry out an algorithms development and optimization [10], $[13,14]$ without having the hardware implementation. Thus, there still a huge gap for further research in reviewing reconfigurable hardware concerning on 3-D transform for medical image compression applications. Two major limitations of the existing works are identified as follows:

a) The image compression has been extensively exploited in $[4,6,15]$. However, medical image compression especially dealing with 3-D modalities is considered as a pre-mature research area.

b) Surveying the literature, even though the family of Daubechies wavelet transform has been used widely in the 3-D DWT implementation [10, 13]. But, there is a small amount that makes use of Daubechies 4tap (Daub4) and 6-tap (Daub6) in the 3-D DWT implementation and thus requires further exploitation.

\section{MATHEMATICAL BACKGROUND FOR DAUBECHIES WAVELET TRANSFORM}

Each of the wavelet transform has their own algorithms includes the scaling and wavelet functions. The only different between the types of wavelet transform is how the algorithms are computed. Daubechies wavelet transform is defined by computing the running averages and differences via scalar products. Besides have properties of longer supports, Daubechies wavelet transform also offers compact support properties. The smaller number of wavelet tap can be used to avoid the edge problem [10]. Thus, the Daubechies 4-tap (Daub4) and 6-tap (Daub6) are used due to their algorithms simplicity compared to others Daubechies family. 


\subsection{Daub4 Algorithm}

The Daub4 wavelet is the simplest wavelet among the Daubechies wavelet families. Generally, Daub4 have four scaling signals and wavelets coefficients as given in (1) and (2) respectively.

$$
\begin{aligned}
& h_{0}=\frac{1+\sqrt{3}}{4 \sqrt{2}}, h_{1}=\frac{3+\sqrt{3}}{4 \sqrt{2}}, h_{2}=\frac{3-\sqrt{3}}{4 \sqrt{2}}, h_{3}=\frac{1-\sqrt{3}}{4 \sqrt{2}} \\
& g_{0}=h_{3}, g_{1}=-h_{2}, g_{2}=h_{1}, g_{3}=-h_{0}
\end{aligned}
$$

The scaling and wavelet functions are calculated by taking the inner product of the coefficients and input data values. In the last iteration, input data of $s[N]$ and $s[N+1]$ does not exist. In other words, the scaling coefficients, $\left(h_{0}, h_{1}, h_{2}, h_{3}\right)$ and wavelet coefficients, $\left(g_{0}, g_{1}, g_{2}, g_{3}\right)$ have length 4 and it would send the $\left(h_{2}, h_{3}\right),\left(g_{2}, g_{3}\right)$ beyond the end of the array of the input signal. This situation is known as edge problem, which will occur in any Daubechies wavelet families.

\subsection{Daub6 Algorithm}

The Daub6 wavelet is the most localized members among Daubechies wavelet families and it has six scaling signals and wavelets coefficients as given in (3) and (4) respectively, where $z_{1}=\sqrt{10}$ and $z_{2}=\sqrt{5+2 \sqrt{10}}$.

$$
\begin{aligned}
& h_{0}=\frac{1+z_{1}+z_{2}}{16 \sqrt{2}}, h_{1}=\frac{5+z_{1}+3 z_{2}}{16 \sqrt{2}}, h_{2}=\frac{10-2 z_{1}+2 z_{2}}{16 \sqrt{2}}, \\
& h_{3}=\frac{10-2 z_{1}-2 z_{2}}{16 \sqrt{2}}, h_{4}=\frac{5+z_{1}-3 z_{2}}{16 \sqrt{2}}, h_{5}=\frac{1+z_{1}-z_{2}}{16 \sqrt{2}} \\
& g_{0}=h_{5}, g_{1}=-h_{4}, g_{2}=h_{3}, g_{3}=-h_{2}, g_{4}=h_{1}, g_{5}=-h_{0}
\end{aligned}
$$

The 1-level Daub6 scaling and wavelet functions are defined in the same way as Daub4 wavelet transform. In the last iteration, the scaling signals and wavelet coefficients of $\left(h_{2}, h_{3}, h_{4}, h_{5}\right)$ and $\left(g_{2}, g_{3}, g_{4}, g_{5}\right)$ respectively would be sent beyond the end of the array. As Daub4 wavelet transform, for handling the edge problem, the data set is treated as it is periodic.

\section{RESULTS AND FINDINGS}

The proposed architectures are concerned on the transformation algorithm for medical image compression applications. To reduce the complexity of the hardware requirements, the 3-D Daub4/Daub6 architecture is divided into three 1-D Daub4/Daub6 with two transpose modules in between.

The purpose of the transpose module is to provide an output in a different order of the input data. Thus, the uses of three 1-D Daub4/Daub6 that are performs the algorithm computations along the rows, columns and $\mathrm{N}$ sub-images respectively can be eliminated to become one operation. So, the 1-D Daub4/Daun6 will perform only one same operation.

\subsection{FPGA-based Daub4 Architecture}

Figure 1 depicts the 1-D Daub4 flow diagram with $N$-inputs sample for pipelined direct mapping implementation. It includes multipliers, shifters, registers and adders for their operation, with notation of 'Mul.', 'Shift.' and 'Add.' for multiplier, shifter and adder, respectively. Since Daubechies wavelet family introduces the edge problem, the shifter is used to wrap-around the input data to the beginning. Moreover, the computation of the Daub4 algorithms requires eight multipliers at each stage to compute the inner product between the input data and Daub4 wavelet coefficients. Each multiplier has a fixed wavelet and scaling function coefficients that will be multiplied with the input data sample. On the other side, the adder with two inputs is used to calculate the summation of the inner products. 
$N$-inputs sample - for every single row

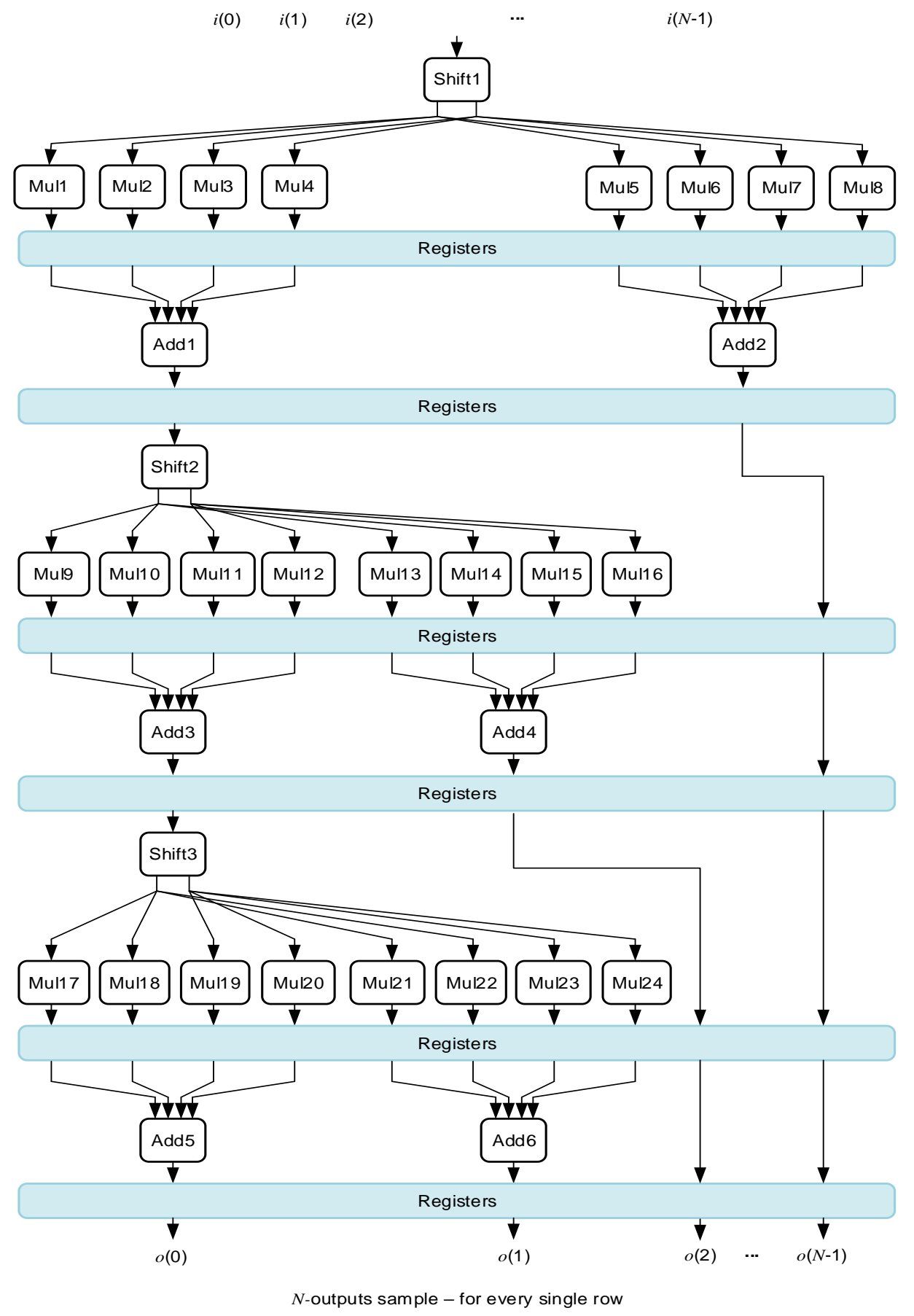

Figure 1. 1-D Daub4 pipelined architecture

\subsection{FPGA-based Daub6 Architecture}

Since the Daub6 algorithm has six scaling and wavelet functions, the 1-D Daub6 flow diagram for pipelined direct mapping implementation requires twelve multipliers at each stage as depicted in Figure 2. Besides the multipliers, the pipelined implementation also uses shifters, adders and registers components. The 1-D Daub6 flow diagram is working in similar way as the 1-D Daub4 flow diagram, where the input data is firstly shifted to avoid the edge problem. After that, the input data is multiplied with the scaling and wavelet function coefficients before the adders calculate the total inner product computation at that stage. 


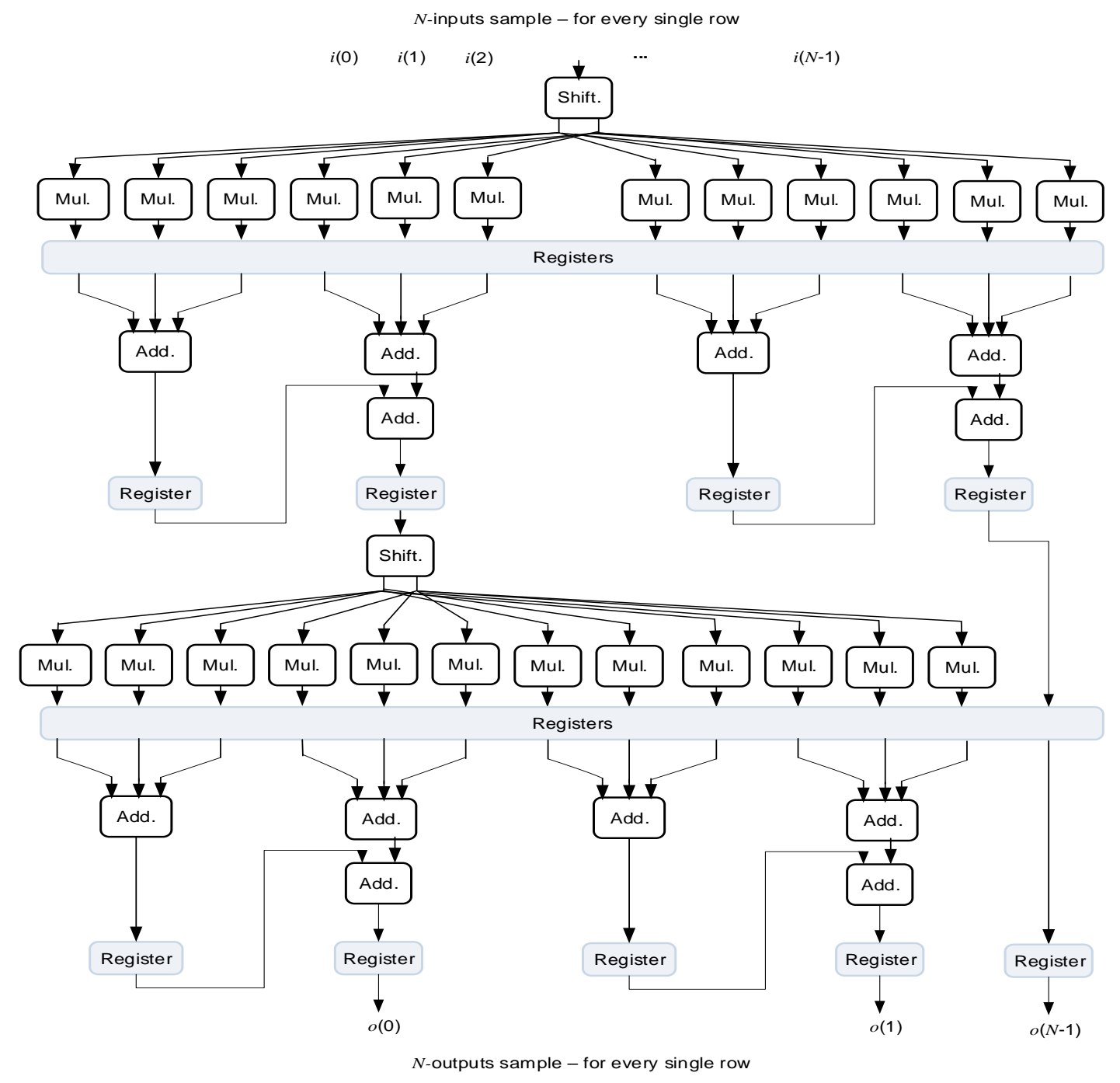

Figure 2. 1-D Daub6 pipelined architecture

\subsection{Implementation Results}

Two proposed architectures were synthesized using VHDL and implemented on Xilinx FPGA single board RIO (sbRIO 9632). Three parameters are selected to evaluate the performance of the proposed pipelined architectures which are area (slices), maximum frequency (MHz) and power consumption (mW) are carried out. Table 1 summaries the implementation results for Daub4 and Daub6 pipelined architectures.

Table 1. Implementation Results

\begin{tabular}{lcc}
\hline Parameters & Proposed Pipelined & Architectures \\
& Daub4 & Daub6 \\
\hline Area (Slices) & $2,731(13.3 \%)$ & $2,968(14.5 \%)$ \\
Maximum Frequency (MHz) & 36.55 & 33.00 \\
Power Consumption (mW) & 142 & 158 \\
\hline
\end{tabular}

In terms of area, Daub4 architecture requires less area with $142 \mathrm{~mW}$ power consumption at 36.55 $\mathrm{MHz}$ speed. In comparison with Daub6, it can be clearly seen that the Daub4 implementation requires less complicated mapping. This is due to the complex algorithms and edge problem that occurs with Daubechies wavelet transform. Moreover, the operation of multiplication in Daub6 implementation consumes more resources, hence $1.2 \%$ of more area are required for Daub6 and $16 \mathrm{~mW}$ of more power obtained with 33 $\mathrm{MHz}$ clock frequency. 
Eventhough Daub6 architecture required more area, it have higher vanishing moments that result in better signal approximation. Thus, the uses of Daub6 wavelet filter in the proposed architecture yields better reconstruction quality for CT image. Figure 3 shows the implementation results for the proposed architectures using CT image.

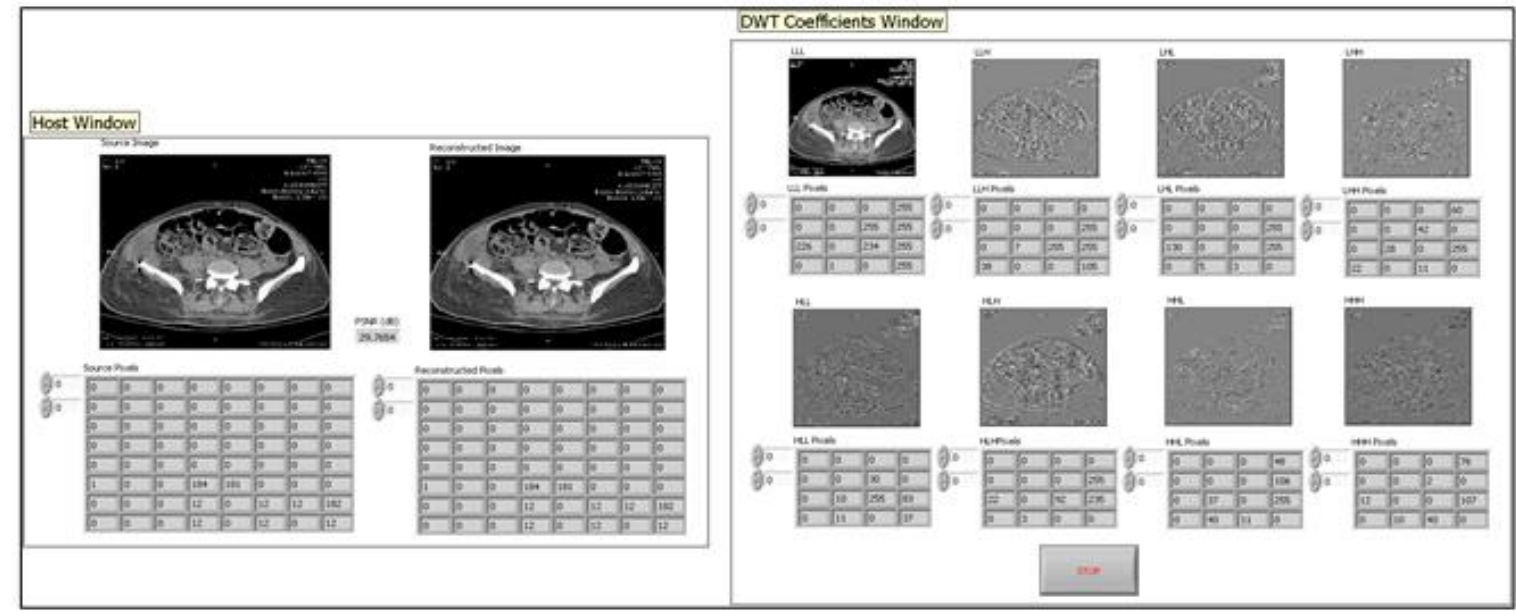

Figure 3. Implementation results with CT image

\section{CONCLUSION}

Two architectures for 3-D Daub4 and Daub6 have been proposed in this paper based on transpose computation for transform block of medical image compression. In addition, the 3-D medical imaging modalities, CT image is used as the input for the compression system.

Comparative study for the proposed architectures has reveals that Daub4 wavelet filter provides better achievements in terms of implementation results. In the other side, Daub6 wavelet filter gives better performance to the reconstruction quality for CT image. On-going research is focusing on the design and FPGA implementation of 3-D DWT using various types of wavelet filters and different design strategies.

\section{REFERENCES}

[1] S. Safie, et al, "Bipolar pulse active features of ECG biometric application," International Conference on Biosignal Analysis, Processing and System (ICBAPS). 2015.

[2] S. Safie, et al, "Comparison of pulse active (PA) modulation signal for electrocardiogram (ECG) authentication," IEEE International Conference on Signal and Image Processing (ICSIPA). 2015, pp. 165-168.

[3] S. S. Mazlan, et al, "Development of anterior cruciate ligament (ACL) knee injury classification system from magnetic resonance image (MRI) using fuzzy inference system," Science and Engineering Technology National Conference (SETNC). 2015.

[4] C. Kamargaonkar and M. Sharma, "Hybrid medical image compression method using sphit algorithm and haar wavelet transform, " International Conference on Electrical, Electronics, and Optimization Techniques (ICEEOT). 2016, pp. $897-900$.

[5] M. G. Ravichandran and A. Dhivakar, "Performance analysis of three-dimensional medical image compression based on discrete wavelet transform," 22nd International Conference on Virtual System and Multimedia (VSMM). 2016, pp. 1-8.

[6] E. Imam and G. Abdalla, "Design and implementation of discrete cosine transform algorithm on fpga device," Conference of Basic Sciences and Engineering Studies (SGCAC). 2016, pp. 13-18.

[7] A. H. Saad and M. Z. Abdullah, "Real-time implementation of fractal image compression in low cost fpga," IEEE International Conference on Imaging Systems and Techniques (IST). 2016, pp. 13-18.

[8] S. Sani, "A wavelet based solar radiation prediction in Nigeria using adaptive neuro-fuzzy approach," Indonesian Journal of Electrical Engineering and Computer Sciences, vol. 12, no 3, December 2018.

[9] H. L. Man and Y. S. Soo, "Modified Pixels Based Fast Median Filter in Impulse Noise Environments," Indonesian Journal of Electrical Engineering and Computer Sciences, vol. 9, no 3, March 2018.

[10] R. D. Reddy, et al, "Lossless compression of medical images for better diagnosis," IEEE 6th International Conference on Advanced Computing (IACC). 2016.

[11] R. Pizzolante, et al, "Parallel low-complexity lossless coding of three-dimensional medical images," 17th International Conference on Network-Based Information Systems. 2014, pp. 91-98. 
[12] D. D. Panda and D. Swain, "Competency assessment of image compression in the lossy and lossless domain," Fourth International Conference on Image Information Processing (ICIIP). 2017, pp. 1-4.

[13] A. Dhivakar, et al, "Medical image compression using embedded zerotree wavelet (ezw) coder," International Conference System Modeling \& Advancement in Research Trends (SMART). 2016.

[14] U. Patbhaje, et al, "A compression system of medical image using wavelet based sparsification and coding," 4th International Conference on Signal Processing and Integrated Networks (SPIN). 2017.

[15] Y. Li, et al, "Curvelet based image compression via vector machine," Optik, 2013, 124, pp. 4859-4866.

\section{BIOGRAPHIES OF AUTHORS}

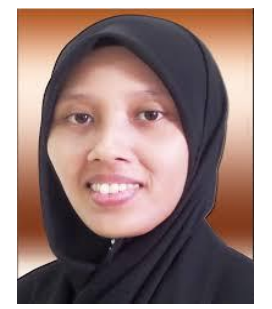

Noor Huda Ja'afar received her B. Eng and M. Eng in Electrical Engineering from Universiti Tun Hussein Onn Malaysia (UTHM) in 2010 and 2013, respectively. She worked as a lecturer at Instrumentation and Control Engineering Section, Universiti Kuala Lumpur Malaysian Institute of Industrial Technology. Currently, she is a Ph.D candidate in the Electrical Engineering, UTHM on the efficient reconfigurable architectures of curvelet transform for 3D medical image compression. Her research interest includes 3D transform, medical imaging and reconfigurable computing.

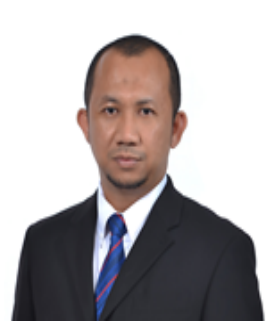

Afandi Ahmad has recently be appointed as Deputy Vice Chancellor of student's affair and alumni in Universiti Tun Hussein Onn Malaysia (UTHM). Currently, he is a senior lecturer at UTHM under Faculty of Electrical and Electronic Engineering. He received his Ph.D. in Electronic and Computer Engineering at Brunel University, London in 2010. He has been awarded a number of grants from government and has published over 130 publications during his career to date. He is a member of IEEE, IAENG, IET and BEM. His research interests include: embedded systems, reconfigurable computing, image processing, medical applications. 